\title{
CANONICAL FORMALISM FOR DESCRIPTION OF CRITICAL PHENOMENA IN SYSTEMS, ISOMORPHIC TO SIMPLE LIQUIDS
}

\author{
V.L. KoulinskiI, N.P.Malomuzh \\ Department of Theoretical Physics, \\ Odessa State University \\ 2 Petra Velykogo St., UA-270100 Odessa, Ukraine
}

Received November 13, 1996; revized January 8, 1997

\begin{abstract}
The basic elements of the canonical formalism for description of the critical phenomena in systems isomorphic to Ising model are stated. The reduction of the effective Hamiltonian to the canonical form and numerous consequences of such a transformation are thoroughly investigated. The shift of fixed point and the critical exponents of the system are considered.
\end{abstract}

\section{Introduction}

At the present time there exists the well-developed approach to the critical phenomena in different systems [1]. All most essential features of the critical behaviour were explained qualitatively and to a certain extent quantitatively $[1,2]$. Especially important role in the investigation of the fluctuation properties belongs to the Renormalization Group (RG) method [3,4]. It allows to describe in an adequate way the symmetry properties of fluctuations as well as to develop the analytical method for calculation of the critical exponents, the universal ratios of critical amplitudes etc.. But in some aspects RG method is not quite consecutive. The critical exponents can be obtained with accuracy $o\left(\epsilon^{2}\right)$ basing on the Landau-Ginsburg Hamiltonian (" $\phi$ ")-model) To calculate the higher terms in the $\epsilon$-expansions the certain extension of the Hamiltonian should be done. The Hamiltonian must be completed with contributions $g_{6} \phi^{6}$ for calculation of $\epsilon^{3}$-order and with $g_{8} \phi^{8}$ for $\epsilon^{4}$-order and so on $[4,1]$. However, this circumstance is not consistent with the assumption that the model " $\phi$ " is sufficient for the description of the long-range fluctuations near critical point. Add, that the Quantum Field Theory analysis of critical anomalies is namely based on " $\phi$ "'-model [6]. This fact can be considered as a property of sufficiency and completeness of that model for that kind of phenomena. The certain difficulty of the standard variant of RG approach is its phenomenological character. The dependence of the Landau-Ginsburg Hamiltonian on the details of microscopic interactions does not ascertained. As a consequence the fine details of the critical behaviour of systems which belong to the same class of universality can not be taken into account. To a certain extent this deficiency can be made up with help of Hubbard-Stratonovich identity for lattice systems [7] or collective variables approach $[9,10]$. But the problems raised by the 
operating with infinite series have the same acuity as the mentioned above. In addition the inclusion of isomorphism principle for the critical phenomena in the multicomponent mixtures into general Hamiltonian formalism remains unsolved problem.

The solution of these problems and other questions can be found in the framework of the canonical formalism. This approach is a synthesis of ideas and methods of the scale-invariant fluctuation theory [1], the catastrophe theory [5] and the statistical theory of condensed matter [11]. Some elements of the canonical formalism were discussed in [14-16].

The central part of such approach is a nonlinear analytical transformation - the canonical transformation of an order parameter that reduces the initial Hamiltonian to polynomial form, so called, canonical form. Its form depends on only the structure of the equations which define the critical point. The list of canonical forms is given in [5].

In this paper the basic ideas of canonical formalism for description of the critical phenomena are formulated. In the first two sections the details of transformation of the effective Hamiltonian to the canonical form in the thermodynamic and the fluctuation regions are discussed. In the third section the renormalization of the parameters of the system caused by the canonical transformation is discussed in the cases of 3-D Ising model and liquid $\mathrm{Ar}$ and $\mathrm{Xe}$ as an example. Section four includes the results obtained in the framework of the canonical RG. The fifth section is devoted to the problem of rectilinear diameter singularity and the analysis of the manifestation of individual features of the system in its critical behaviour. Some new problems and possible applications of the canonical formalism are enumerated in Conclusion.

\section{Effective Hamiltonian in thermodynamic region}

Let $h=h(\phi, T)$ and $F=F(\phi, T)$ are the equation of state and the free energy of a system, respectively:

$$
h=\left.\frac{\partial F}{\partial \phi}\right|_{T},
$$

where $T$ - the temperature, $h$ - the external field conjugated to the order parameter $\phi$. In the vicinity of the critical point defined by the equations:

$$
\frac{\partial h}{\partial \phi}=0, \quad \frac{\partial^{2} h}{\partial \phi^{2}}=0,
$$

the free energy of a system can be represented as following:

$$
-\beta F(\phi, T)=\sum_{k=1}^{\infty} \frac{\lambda_{k}}{k} \phi^{k}, \quad \beta=\frac{1}{k_{\mathrm{B}} T} .
$$

To determine coefficients $\lambda_{k}$ it is expedient to use the equation of state:

$$
\lambda_{k}=\left.\frac{1}{(k-1) !} \frac{\partial^{k-1} h}{\partial \phi^{k-1}}\right|_{\phi=0}, \quad k=1,2, \ldots
$$

For example, in the case of Curie-Weiss equation for magnetics:

$$
\beta h=-\beta \tilde{J}(0) m+\operatorname{atanh} m, \quad \phi \equiv m,
$$


where $m$ and $h$ are magnetization and external field strength, respectively,

$$
\tilde{J}(\mathbf{k})=\sum_{i, j} J\left(\mathbf{r}_{i j}\right) \exp \left(i \mathbf{k r}_{i j}\right)
$$

is Fourier transform of the "spin" interaction potential, the coefficients $\lambda_{k}$ have the form:

$$
\lambda_{1}=h, \quad \lambda_{2}=\beta \tilde{J}(0)-1, \quad \lambda_{2 k-1}=0, \quad \lambda_{2 k}=-\frac{1}{2 k-1}, k \geqslant 2 .
$$

Further we will consider the critical behaviour of simple liquids basing on two model equations of state. In this case we take the well-known van der Waals equation (vdW) :

$$
p=\frac{n k_{\mathrm{B}} T}{1-n b}-n^{2} a
$$

and modified van der Waals equation $(\operatorname{mvd} W)$ :

$$
p=p_{0}-a n^{2}+c n^{3},
$$

where

$$
p_{0}=n k_{\mathrm{B}} T \frac{1+\eta+\eta^{2}+\eta^{3}}{(1-\eta)^{3}}
$$

is a hard core pressure term in Carnahan-Starling approximation [8], $\eta=\frac{\pi n \sigma^{3}}{6}$ is dimensionless density, $\sigma$ is hard core diameter. The expressions for the coefficients $a$ and $c$ will be given below. If the state of system is described by $\phi=\tilde{v}-1$, where $\tilde{v}=\frac{n_{c}}{n}, n_{c}$ is the critical density, than for coefficients $\lambda_{k}$ we get from equation (7):

$$
\lambda_{1}=\tilde{P}-1, \quad \lambda_{k}=(-1)^{k} 4\left(\frac{3}{2}\right)^{k-1}\left(\frac{\alpha_{k}-\tau}{1-\tau}\right), k=1,2, \ldots,
$$

where

$$
\alpha_{k}=\frac{3 k}{4}\left(\frac{2}{3}\right)^{k-1}-1
$$

Here $\tau=\tilde{T}-1, \tilde{T}=T / T_{c}, \tilde{P}=P / P_{c}$. The values of the critical temperature, pressure and density are:

$$
T_{c}=\frac{8 a}{27 c}, \quad P_{c}=\frac{a}{27 b^{2}}, \quad n_{c}=\frac{1}{3 b} .
$$

For mvdW equation it is convenient to take variable $\phi$ in a form $\phi=u-u_{c}$, where $u=1 / \eta$. For this case we obtain from equations (8) and (9)

$$
\begin{gathered}
\lambda_{k}=(-1)^{k} \frac{32 \tilde{T} b_{k}-c_{k}}{\tilde{T}}-P_{c} \delta_{k, 1}, \\
b_{k}=\frac{k(k+1)}{\left(u_{c}-1\right)^{k+2}}+\frac{4 k}{\left(u_{c}-1\right)^{k+1}}+\frac{1}{u_{c}^{k}},
\end{gathered}
$$




$$
c_{k}=\frac{432 k(1+2(k+1) c / a b)}{u_{c}^{k+1}} .
$$

Obviously the external field is $\lambda_{1}=\hat{P}-\hat{P}_{c}$. The critical phenomena is observed at $\lambda_{1}=0$ near the point determined by equations:

$$
\lambda_{2}=0, \quad \lambda_{3}=0 .
$$

At the same time the condition $\lambda_{4}<0$ takes place in all considered examples. Reducing of the infinite series (3) to the polynomial canonical form is the fact of great importance. The mathematical for such a procedure are discussed in the catastrophe theory [5]. With the help of the canonical transformation of the initial order parameter

$$
\eta \longrightarrow \phi \equiv \hat{C}_{g} \eta=-\alpha_{0}+\eta+\frac{1}{2} \alpha_{2} \eta^{2}+\frac{1}{3} \alpha_{3} \eta^{3}+\ldots,
$$

equation (3) can be reduced to the canonical form which is the same as Landau free energy:

$$
\delta F(\phi)=V\left[-h_{\phi} \phi+\frac{1}{2} a_{2}^{c} \phi^{2}+\frac{1}{4} a_{4}^{c} \phi^{4}\right] .
$$

One can show that

$$
\begin{gathered}
h_{\phi}=\lambda_{1}+\frac{1}{3 \lambda_{4}} \lambda_{2} \lambda_{3}-\frac{2 \lambda_{3}^{3}}{27 \lambda_{4}^{2}}, \\
a_{2}^{c}=\lambda_{2}-\frac{1}{3} \frac{\lambda_{3}^{2}}{\lambda_{4}}, \\
a_{4}^{c}=\lambda_{4},
\end{gathered}
$$

where

$$
\begin{gathered}
\lambda_{1}=h_{\eta}, \\
\lambda_{2}=A_{2}-h_{\eta} \gamma_{2}, \\
\lambda_{3}=A_{3}+\frac{3}{2} A_{2} \gamma_{2}-h_{\eta} \gamma_{3}, \\
\lambda_{4}=A_{4}+\frac{2}{3} A_{3} \gamma_{2}+A_{2}\left(\gamma_{2}^{2}+\frac{4}{3} \gamma_{3}\right)-h_{\eta} \gamma_{4}
\end{gathered}
$$

and $\gamma_{n}$ are the coefficients of the transformation inverse to (14):

$$
\eta \equiv \hat{C} \phi=-\gamma_{0}+\phi+\frac{1}{2} \gamma_{2} \phi^{2}+\frac{1}{3} \gamma_{3} \phi^{3}+\ldots
$$

Taking into account that parameters $x_{i}=\left(h_{\eta}, A_{2}, A_{3}\right)$ are small near the critical point the coefficients can be represented in a form:

$$
\gamma_{n}=\gamma_{n}^{(0)}+\gamma_{n}^{(i)} x_{i}+\gamma_{n}^{(i, j)} x_{i} x_{j}+\ldots,
$$

where

$$
\gamma_{(0)}=-\alpha_{0}, \quad \gamma_{2}^{(0)}=-\frac{2}{5} \frac{A_{5}}{A_{4}}, \quad \gamma_{3}^{(0)}=-\frac{1}{2} \frac{A_{6}}{A_{4}}-\frac{3}{4} \gamma_{2}(0)^{2},
$$




$$
\begin{gathered}
\gamma_{4}^{(0)}=-\frac{4}{7} \frac{A_{7}}{A_{4}}+\frac{16}{3} \gamma_{3}^{(0)} \gamma_{2}^{(0)}-\frac{15}{3} \gamma_{2}^{(0)^{3}} \\
\gamma_{2}^{(1)}=-\frac{\gamma_{5}^{(0)}}{A_{4}}, \quad \gamma_{3}^{(1)}=-\frac{21}{4} \gamma_{2}^{(1)} \gamma_{2}^{(0)}-\frac{1}{2} \frac{\gamma_{6}^{(0)}}{A_{4}} \quad \text { etc. }
\end{gathered}
$$

The formulas (16) can be considered as a result of canonical transformation in the space of the Hamiltonian coefficients. From the physical point of view the canonical transformation is connected with the renormalization of the initial order parameter. This transformation generalizes the notation of the temperature and the external field conjugated to the order parameter. Due to canonical transformation the order parameter acquires rigorous physical meaning. It is important to mark that the roots of the equations

$$
a_{1}^{c}=0, \quad a_{2}^{c}=0
$$

and those of (11) or (13) are the same. That is because of spatial homogeneity of both the initial and canonical order parameters. At the same time it is clear that only the change in the weight of short scale fluctuations influences the critical point position.

At the end of this section we touch upon the question of the convergence of used expansions. The convergence radius $R$ for the series of the free energy (3) depends on both the choice of the order parameter and the equation of state. In the examples mentioned above:

$$
R=\left\{\begin{array}{cll}
1 & , \text { Ising model (Curie-Weiss equation) } \\
\frac{1}{3} & , \text { vdW equation } \\
4.75 & , \text { mdV equation }
\end{array}\right.
$$

The radii of convergency for the expansions (14), (18) and (19) as it follows from the results of [5], are less than those given in (22).

\section{Effective Hamiltonian in the fluctuation region}

The situation changes essentially when the state of system belongs to the fluctuation region. Here the spatial inhomogeneity of the order parameter should be taken into consideration. The behaviour of fluctuations is described by the partition function:

$$
Z=\int \exp \left[-H_{l}[\eta(\vec{r})]-H_{q l}[\eta(\vec{r})]\right] D \eta(\vec{r}),
$$

in which

$$
\begin{gathered}
H_{l}[\psi(\vec{r})]=\beta \int \mathcal{H}_{l}^{(0)}(\eta(\vec{r})) \mathrm{d} V, \\
\mathcal{H}_{l}^{(0)}(\eta(\vec{r}))=\sum_{n=1}^{\infty} \frac{1}{n} A_{n} \eta^{n}(\vec{r}), \quad A_{1}=h_{\eta} . \\
H_{q l}[\eta(\vec{r})]=\beta \frac{b}{2} \int(\nabla \eta(r))^{2} \mathrm{~d} V,
\end{gathered}
$$

$\beta=\frac{1}{k_{\mathrm{B}} T}, T$ is the temperature, $k_{\mathrm{B}}$-Boltzmann's constant. One should note that local and nonlocal contributions influence the thermodynamical 
properties and the character of fluctuations in a different way. The thermodynamical properties are determined by the local terms and the longrange correlations are described mainly by the quasilocal approximation $[1,10]$. The coefficients $A_{n}$ are the same as those in (3). In consequence of spatial inhomogeneity the canonical transformation (14) can be applied to $H_{l}^{(0)}[\eta(\vec{r})]$ only locally. As a result, $\mathcal{H}_{l}^{0}[\eta(\vec{r})]$ transforms into

$$
\mathcal{H}_{l}^{(1)}\left[\phi_{1}(\vec{r})\right]=\mathcal{H}_{l}^{(0)}\left(\phi_{1}(\vec{r})\right)-\frac{1}{\beta} \ln J_{1,0}\left[\phi_{1}(\vec{r})\right],
$$

where $J_{1,0}$ is the Jacobian of the transformation from $\eta(\vec{r})$ to $\phi_{1}(\vec{r})=\hat{C} \eta(\vec{r})$ Though $\mathcal{H}_{l}^{(0)}\left[\phi_{1}(\vec{r})\right]$ takes the canonical form

$$
\mathcal{H}_{l}^{(0)}\left(\phi_{1}(\vec{r})\right)=-h_{\phi_{1}}\left[\phi_{1}(\vec{r})\right]+\frac{1}{2} a_{2}^{c}(1) \phi_{1}^{2}(\vec{r})+\frac{1}{4} a_{4}^{c}(1) \phi_{1}^{4}(\vec{r}),
$$

with coefficients determined by (16)-(20); due to the Jacobian term in (26) the infinite series of local contributions appears again. The quasilocal part $H_{q l}[\eta(\vec{r})]$ of the fluctuation Hamiltonian remains unchanged if we restrict ourselves only by quadratic term:

$$
H_{q l}[\eta(\vec{r})] \rightarrow H_{q l}\left[\phi_{1}(\vec{r})\right]
$$

Therefore

$$
Z[\eta(\vec{r})]=Z[\hat{C} \eta(\vec{r})]=\int \exp \left(-H_{l}^{(1)}\left[\phi_{1}(\vec{r})\right]-H_{q l}\left[\phi_{1}(\vec{r})\right]\right) D \phi_{1}(\vec{r}) .
$$

If we continue this procedure we obtain on the $i$-th step $\left(\phi_{0}(\vec{r}) \equiv \eta(\vec{r})\right)$ :

$$
Z[\eta(\vec{r})]=Z[\hat{C} \ldots \hat{C} \eta(\vec{r})]=\int \exp \left(-H_{l}^{(i)}\left[\phi_{i}(\vec{r})\right]-H_{q l}\left[\phi_{i}(\vec{r})\right]\right) D \phi_{i}(\vec{r}),
$$

where

$$
\begin{gathered}
H_{l}^{(i)}=\beta \int \mathcal{H}_{l}^{(i)}\left[\phi_{i}(\vec{r})\right] \mathrm{d} V, \\
\mathcal{H}_{l}^{(i)}\left(\phi_{i}(\vec{r})\right)=\mathcal{H}_{l}^{(i-1)}\left(\phi_{i}(\vec{r})\right)-\frac{1}{\beta} \ln J_{i, i-1}\left[\phi_{i}(\vec{r})\right],
\end{gathered}
$$

and $\mathcal{H}_{l}^{(i-1)}\left[\phi_{i}(\vec{r})\right]$ similar to $\mathcal{H}_{l}^{(0)}$ takes the canonical form. One should note that the recursive procedure is a result of the account of the Jacobian. The convergency properties of the recursion described depends on the the choice of the initial order parameter. For convergent procedure:

$$
\lim _{i \rightarrow \infty} \phi_{i}(\vec{r})=\phi(\vec{r})=\hat{C}_{\infty} \eta(\vec{r}), \quad \hat{C}_{\infty}=\prod_{i=1}^{\infty} \hat{C}(i),
$$

and

$$
\begin{gathered}
\lim _{i \rightarrow \infty} \mathcal{H}_{l}^{(i)}[\phi(\vec{r})] \equiv \mathcal{H}_{l}^{c}[\phi(\vec{r})]=\hat{C}_{\infty} \mathcal{H}_{l}^{(0)}[\eta(\vec{r})]= \\
=-h_{\phi} \phi(\vec{r})+\frac{1}{2} a_{2}^{c} \phi^{2}(\vec{r})+\frac{1}{4} a_{4}^{c} \phi^{4}(\vec{r}) .
\end{gathered}
$$


The formulas (32) and (33) give the definition of the canonical transformation operator in fluctuation region. To get the explicit expressions for the coefficients $h_{\phi}, a_{2}^{c}$ and $a_{4}^{c}$ with necessary accuracy it is sufficient to describe the algorithm of their constructing

$$
\begin{aligned}
& a_{1}^{c}=\tilde{a}_{1}(\infty)-\frac{\tilde{a}_{2}(\infty) \tilde{a}_{3}(\infty)}{3 \tilde{a}_{4}(\infty)}+\frac{2 \tilde{a}_{3}^{3}(\infty)}{27 a_{4}^{2}(\infty)} \\
& a_{2}^{c}=\tilde{a}_{2}(\infty)-\frac{\tilde{a}_{3}(\infty)}{3 \tilde{a}_{4}(\infty)}, \\
& a_{4}^{c}=\tilde{a}_{4}(\infty) .
\end{aligned}
$$

At every finite step of the transformation we get:

$$
\begin{gathered}
\tilde{a}_{1}(i)=\tilde{a}_{1}(i-1)-\Gamma_{2}(i) \\
\tilde{a}_{2}(i)=\tilde{a}_{2}(i-1)+\tilde{a}_{1}(i-1) \Gamma_{2}-2\left[\Gamma_{3}(i)-\frac{1}{2} \Gamma_{2}^{2}(i)\right], \\
\tilde{a}_{3}(i)=\tilde{a}_{3}(i-1)+\frac{3}{2} \tilde{a}_{2}(i-1) \Gamma_{2}(i)+\tilde{a}_{1}(i-1) \Gamma_{3}(i)- \\
3\left[\Gamma_{4}(i)-\Gamma_{3}(i) \Gamma_{2}(i)+\frac{1}{3} \Gamma_{2}^{3}(i)\right] \\
\tilde{a}_{4}(i)=\tilde{a}_{4}(i-1)+\tilde{a}_{2}(i-1)\left[\Gamma_{2}^{2}(i)+\frac{4}{3} \Gamma_{3}(i)\right]+\tilde{a}_{1}(i) \Gamma_{4}(i)- \\
-4\left[\Gamma_{5}(i)+\Gamma_{4}(i) \Gamma_{2}(i)+\frac{1}{2} \Gamma_{3}^{2}(i)--\Gamma_{3}(i) \Gamma_{2}^{2}(i)-\frac{1}{4} \Gamma_{2}^{4}(i)\right] .
\end{gathered}
$$

Here $\Gamma_{n}(i)$ are the coefficients of the inverse canonical transformation on the $i$-th step of the recursive procedure:

$$
\phi_{i-1}(\vec{r})=\phi_{i}(\vec{r})++\frac{1}{2} \Gamma_{2}(i) \phi_{i}^{2}(\vec{r})+\frac{1}{3} \Gamma_{3}(i) \phi_{i}^{3}(\vec{r})+\ldots
$$

They can be calculated by formulas (19) and (20) in which instead of coefficients $A_{n}$ the coefficients $A_{n}(\tilde{i}-1)$ of expansions of $\mathcal{H}_{l}^{(i-1)}\left[\phi_{i}(\vec{r})\right]$ in series of $\phi_{i}(\vec{r})$ should be substituted. In accordance with (31) we get:

$$
\begin{gathered}
A_{n} \rightarrow \tilde{A}_{n}(i-1)=A_{n}(i-1)+A_{n}^{J}(i-1), \\
A_{n}(i-1)=\sum_{k=1}^{n} \sum_{\sigma} \tilde{A}_{k}(i-2) \frac{\prod_{p=1}^{n} \Gamma_{p}^{\alpha_{p}}(i-1)}{\prod_{p=1}^{n} p^{\alpha_{p}}} \frac{(k-1) !}{\prod_{p=1}^{n} \alpha_{p} !}, \\
A_{n}^{J}(i-1)=\sum_{k=1}^{n} \sum_{\sigma^{\prime}}(-1)^{k} \frac{\prod_{p=2}^{n+1} \Gamma_{p}^{\alpha_{p}}(i-1)}{\prod_{p=1}^{n} p^{\alpha_{p}}} \frac{(k-1) !}{\prod_{p=2}^{n+1} \alpha_{p}} .
\end{gathered}
$$

Here $\sum_{\sigma}$ and $\sum_{\sigma^{\prime}}$ denote the summation over all sets of $\alpha_{n}$ which satisfy the conditions

$$
\sum_{\sigma}:, \quad \sum_{p=1}^{n} \alpha_{p}=k, \quad \sum_{p=1}^{n} p \alpha_{p}=n
$$


and

$$
\sum_{\sigma^{\prime}}:, \quad \sum_{p=2}^{n+1} \alpha_{p}=k, \quad \sum_{p=2}^{n+1} p \alpha_{p}=n+k .
$$

The arguments used can be completed by nonperturbative approach. If we start from the partition function for the local Hamiltonian the existence of the canonical form is equivalent to the existence of such a transformation $\eta \rightarrow \phi$ for which the following conditions hold

$$
\begin{aligned}
& \Phi[+\infty ;]=U\left[+\infty ; a_{1}^{c}, a_{2}^{c}\right] \\
& \Phi[-\infty ;]=U\left[-\infty ; a_{1}^{c}, a_{2}^{c}\right] .
\end{aligned}
$$

Here

$$
\begin{gathered}
\Phi[x]=\int_{0}^{x} \mathrm{~d} \phi \exp \left(-H_{l}[\phi]\right), \\
U\left[y ; a_{1}^{c}, a_{2}^{c}\right]=\int_{0}^{y} \mathrm{~d} \phi \exp \left(-H^{c}[\eta]\right) .
\end{gathered}
$$

The equations (40) determines uniquely the canonical coefficients if the value of $a_{4}^{c}$ is fixed.

It should be pointed out that canonical form of the fluctuation Hamiltonian coincides completely with Landau-Ginsburg Hamiltonian. But the order parameter of the system and the "temperature" $a_{2}^{c}$ conjugated to it as well as the "external" field $a_{1}^{c}$ and also intermodal interaction coefficient $a_{4}^{c}$ are connected with their initial values by nonlinear relations. It is important that all odd terms in canonical form of the Hamiltonian are absent. Note, that the choice of the initial order parameter essentially influence the convergence (or asymptotic character) of the recursive procedure described above. The closer the one is to the canonical order parameter the best convergency to the canonical form can be achieved.

\section{Operator of the canonical RG-transformation}

Let $\hat{R}(\lambda)$ be the operator of RG-transformation. The action of $\hat{R}(\lambda)$ on the Landau-Ginsburg Hamiltonian $H_{L G}$ changes its form:

$$
\hat{R}(\lambda) \circ H_{L G}[\phi(\vec{r})] \rightarrow H_{L G}[\tilde{\phi}(\vec{r})]+\int \mathrm{d} V \sum_{k=5}^{\infty} \frac{\chi_{k}}{k} \tilde{\phi}^{k}(\vec{r})+H_{n l}[\tilde{\phi}(\vec{r})],
$$

where $\tilde{\phi}(\vec{r})=Z(\lambda) \phi(\vec{r}), Z(\lambda)$ is the coefficient of the scaling factor, $H_{n l}$ denotes the sum of nonlocal contributions which are not included in $H_{L G}$. To remove the "superfluous" local and nonlocal terms Wilson [3] completed the definition of RG-operator with projector operator $\hat{P}$ onto the space of Landau-Ginsburg Hamiltonians. In accordance with this

$$
\hat{R}(\lambda) \rightarrow \hat{R}_{W}(\lambda)=\hat{P} \circ \hat{R}(\lambda), \hat{R}_{W}(\lambda) \circ H_{L G}[\phi(\vec{r})] \rightarrow H_{L G}[\tilde{\phi}(\vec{r})] .
$$

One can improve the definition of the RG-operator substituting $\hat{P}$ by the product $\hat{P} \circ \hat{C}_{\infty}$, where the operator of local canonical transformation $\hat{C}_{\text {infty }}$ is given by(32). The new RG-operator

$$
R_{c}=\hat{P} \circ \hat{C} \circ \hat{R}(\lambda)
$$


is the canonical RG-operator. We suppose also that operator $\hat{P}$ kills the contributions of small scale with wave vectors $|\vec{q}|>\Lambda$, where $\Lambda$ is the cut off parameter. Unlike $R_{W}$ the operator $R_{c}$ not only conserves the form of Landau-Ginsburg Hamiltonian:

$$
\hat{R}_{c}(\lambda) \circ H_{G L}[\phi(\vec{r})]=H_{L G}[\tilde{\phi}(\vec{r})],
$$

but also takes into account all local contributions. This circumstance influences essentially on the parameters of RG-transformation near fixed point.

To analyse the properties of RG-transformations we pass from functional equation (44) to the differential equations for the coefficients of LandauGinsburg Hamiltonian. In the limit $t=\ln \lambda \rightarrow 0$ at $a_{1}^{c}=0$ and fixed value of $b$ we obtain the equations:

$$
\begin{aligned}
\frac{d r}{d t} & =f_{2}(r, g) \\
\frac{d g}{d t} & =f_{4}(r, g)
\end{aligned}
$$

in which $r=a_{2}^{c}$ and $g=\frac{1}{4 !} a_{4}^{c}$. The equations (45) correspond to the Gell-Mann-Low equations in quantum field theory [17]. Below we consider their solutions near saddle-like fixed point for local $(b=0)$ and quasilocal Hamiltonians.

\section{Properties of scale transformations for local Hamilto- nian}

The action of RG-operator $\hat{R}(\lambda)$ on local Hamiltonian

$$
H_{l}=\int \mathrm{d} V\left[\frac{1}{2} a_{2} \phi^{2}(\vec{r})+\frac{1}{4} a_{4} \phi^{4}(\vec{r})\right]
$$

leads to the following expressions for its coefficients

$$
\begin{aligned}
& a_{2}(t)=a_{2}+t R_{2}+o(t), \\
& a_{4}(t)=a_{4}+t R_{4}+o(t), \\
& \chi_{2 n}=t R_{2 n}+o(t) .
\end{aligned}
$$

Representing $\Gamma_{2 n+1}=\Gamma_{2 n+1}(t)$ in a form

$$
\Gamma_{2 n+1}(t)=t G_{2 n+1}+o(t), \quad\left(\Gamma_{2 n}=0, n=1,2, \ldots\right)
$$

for the we get coefficients $a_{2}^{c}$ and $a_{4}^{c}$ of the canonical Hamiltonian

$$
\begin{array}{lll}
a_{2}^{c}=a_{2}+t\left(R_{2}-2 G_{3}\right) & +o(t) \\
a_{4}^{c}=a_{4}+t\left(R_{4}-4 ! G_{5}+8 a_{2} G_{3}\right) & +o(t)
\end{array}
$$

One can show that coefficients $G_{3}$ and $G_{5}$ satisfy the equations

$$
G_{3}(p)=\sum_{q=0}^{p} \sigma_{3}(q), \quad G_{5}(p)=\sum_{q=0}^{p} \sigma_{5}(q),
$$

where $\sigma_{n}(q)$ are the coefficients of nonlinear transformation of the order parameter at $q$-th step of recursive procedure. At the same time for $\sigma_{n}(1)$ we have:

$$
R_{2 n}+\frac{4}{2 n-3} g \sigma_{2 n-3}(1)+\frac{1}{2 n+3} r \sigma_{2 n-1}(1)+\ldots=0 .
$$


It follows from equation (51) that:

$$
\sigma_{2 n-3}(1)=\sum_{k=0}^{\infty} c_{2 n-3}^{3}(1) r^{k},
$$

where

$$
c_{2 n-3}^{k}(1)=(-1)^{k+1} \frac{1}{2^{k+1}}\left(\frac{2 n-3}{2}\right)_{k+1} R_{2(n+k)},
$$

and $(a)_{k}=a(a+1) \ldots(a+k-1)$ is Pochgammer symbol. As a consequence of (49)-(53) we get the equations (45) with

$$
\begin{aligned}
f_{2}^{c}= & f_{2}+\sum_{k=0}^{\infty}(-1)^{k} \frac{1}{2^{k+1}}\left(\frac{3}{2}\right)_{k+1} \frac{r^{k}}{g} f_{2(k+3)} . \\
f_{4}^{c}=f_{4} & +\frac{1}{3} \sum_{k=0}^{\infty}(-1)^{k+1} \frac{1}{2^{k+1}}\left(\frac{3}{2}\right)_{k+1} \frac{r^{k+1}}{g} f_{2(k+3)}+ \\
& +\sum_{k=0}^{\infty}(-1)^{k} \frac{1}{2^{k+1}}\left(\frac{5}{2}\right)_{k+1} \frac{r^{k}}{g} f_{2(k+4)} .
\end{aligned}
$$

It is important to note that expansions of $f_{2 n}, n>2$ in seria on $g$ begin with $g^{n}$. In order to calculate the critical exponent $\nu$ for the correlation radius we use the results of [18] taking into account that in the proposed approach the interactions $\phi^{n}, n \geqslant 3$ are absent as well as the contributions in $f_{6}, f_{8}$, etc. depending on the spatial dimension $D$. According [18] the $g$-expansions of $f_{2 n}$ are as following:

$$
\begin{gathered}
f_{2}=\frac{2 r}{D}+3 g\left(1+2 g+24 g^{2}+\ldots\right)+o\left(g^{4}\right), \\
f_{4}=\frac{4-D}{D} g-9 g^{2}-108 g^{3}-2614 g^{4}+o\left(g^{4}\right), \\
f_{6}=36 g^{3}+1296 g^{4}+o\left(g^{4}\right), \\
f_{8}=-162 g^{4}+o\left(g^{4}\right) .
\end{gathered}
$$

As a result of (55) and (54) we obtain

$$
\begin{gathered}
f_{2}^{c}=\frac{2 r}{D}+3 g+6 g^{2}+72 g^{3}+\left\{27 g^{2}+972 g^{3}+\frac{243}{4} r g^{2}\right\}+o\left(g^{3}\right), \\
f_{4}^{c}=\frac{4-D}{D} g-9 g^{2}-108 g^{3}-\left\{\frac{405}{2} g^{3}+18 r g^{2}\right\}+o\left(g^{3}\right),
\end{gathered}
$$

where the terms in braces are the contributions caused by the canonical transformations. The position of the nontrivial fixed point $\left(r^{*}, g^{*}\right)$ is determined by equations

$$
\begin{gathered}
r^{*}=-\frac{3 D}{2} g^{*}+o\left(g^{*}\right), \\
\frac{4-D}{D}-9 g^{*}-\left(27 D+\frac{621}{2}\right) g^{*^{2}}+o\left(g^{*^{2}}\right)=0 .
\end{gathered}
$$


The matrix elements of the canonical RG-operator near fixed point are equal to

$$
\begin{array}{lll}
\left(\hat{R}_{c}\right)_{11}=2-3 D g^{*}\left[1+6 g^{*}+\left\{\frac{27}{4} g^{*}\right\}\right] & +o\left(g^{*^{2}}\right), \\
\left(\hat{R}_{c}\right)_{12}=\frac{D}{4}\left[\left(1+4 g^{*}+72 g^{*^{2}}\right)+\left\{18 g^{*}+\left(972-\frac{81 D}{2}\right) g^{*^{2}}\right\}\right] & +o\left(g^{*^{2}}\right), \\
\left(\hat{R}_{c}\right)_{21}=18 D g^{*^{2}}+\left\{-18 D g^{*^{2}}\right\} & +o\left(g^{*^{2}}\right), \\
\left(\hat{R}_{c}\right)_{22}=4-D-18 D g^{*}-324 g^{*^{2}}+3\left\{18 D-\frac{405}{2}\right\} g^{*^{2}} & +o\left(g^{*^{2}}\right) .
\end{array}
$$

Note that for the calculation of the matrix elements with accuracy $o\left(g^{2}\right)$ the parameter $r^{*}$ should be calculated in linear approximation $[12,1]$ The value of the critical exponent $\nu$ of the correlation radius that corresponds to (58) is:

$$
\nu=\frac{1}{2}\left[1+\frac{3 D}{2} g^{*}+\frac{9 D}{4} g^{*^{2}}+\left\{\frac{17}{2} g^{*^{2}}\right\}\right]+o\left(g^{*^{2}}\right) .
$$

The contributions appeared due to the canonical transformation are given in braces. The values of coordinates of the fixed point and the correlation radius critical exponent are presented in table 1. The comparison of the

Table 1. Correlation radius exponent and fixed point coordinates.

\begin{tabular}{|c|c|c|c|}
\hline & $r^{*}$ & $g^{*}$ & $\nu$ \\
\hline $\mathrm{D}=2$ & -0.142 & 0.047 & 0.74 \\
\hline $\mathrm{D}=3$ & -0.105 & 0.023 & 0.56 \\
\hline
\end{tabular}

results with those obtained numerically in [18] shows that the value of $g^{*}$ is very close to that in [18]. At the same time the value of critical exponent $\nu$ obtained by us is greater than the analogous value $(\nu=0.54069)$ calculated for the Hamiltonian (46) in $g^{3}$-approximation but less than the one $(\nu=$ $0.60443)$ for the Hamiltonian:

$$
H_{l}=\int \mathrm{d} V\left[\frac{1}{2} a_{2} \phi^{2}(\vec{r})+\frac{1}{4} a_{4} \phi^{4}(\vec{r})+\frac{1}{6} a_{6} \phi(\vec{r})\right] .
$$

It should be noted that in canonical renormgroup approach there is no need to change the model for calculations of $g$-expansions in higher orders.

\section{The values of the parameters in the critical point}

In this section we give the values of the several important parameters for 3-D Ising model and liquid Ar and Xe obtained in the framework of the canonical formalism. First of all we should determine the critical point position. In accordance with previous sections the values of critical temperature and the external field are connected with their initial values by the following relations:

$$
T_{c}=T_{0}\left(1+\tau^{*}\right), \quad h_{c}=h_{0}+h^{*},
$$

where $\tau^{*}$ and $h^{*}$ are determined by the equations:

$$
a_{1}^{c}\left(\tau^{*}, h^{*}\right)=0, \quad a_{2}^{c}\left(\tau^{*}, h^{*}\right)=0 .
$$


This is the system of nonlinear equations which has in general multiple solutions. The physical ones are those $\left(\tau^{*}, h^{*}\right), i=1, \ldots, n$ which belong to the convergence region and satisfy the stability conditions:

$$
a_{4}^{c}\left(\tau^{*}, h^{*}\right)<0 .
$$

If still $i>1$ then the solution which is our "analytic prolongation" of the initial critical point should be chosen. The equations (62) have the simplest form in the case of Ising model. The coefficient $a_{1}^{c}$ does not depend on $\tau$ and the condition of its vanishing yields to $h^{*}=0$ which agrees with the symmetry of the Ising model. The second one of the equations (62) expanded in series on $\tau$ is as following:

$$
2 \Gamma_{3}^{(0)}+\left(1+2 \Gamma^{(2)}\right) \tau^{*}+\Gamma_{3}^{(2,2)} \tau^{*^{2}}+\ldots=0 .
$$

Using the expressions (6) for coefficients $\lambda_{k}$ after first iteration (see Section 3) we get:

$$
\Gamma_{3}^{(0)} \simeq-0.3, \quad \Gamma_{3}^{(2)} \simeq-0.03, \quad \ldots
$$

The root $\tau^{*}$ of (64) equation is approximately equal to:

$$
\tau^{*}=0.53 .
$$

Since $T_{0}=z J / k_{\mathrm{B}}$, where $z=z(D)$ is the coordination number, the equations (62) and (66) yield the following value of the critical temperature for "cubic" Ising model:

$$
k_{\mathrm{B}} T_{c}=\left\{\begin{array}{ccc}
\frac{J}{0.26}+o\left(\tau^{*}\right) & , \quad D=3, \\
\frac{J}{0.39}+o\left(\tau^{*}\right), & D=2 .
\end{array}\right.
$$

High temperature expansion for $D=3$ case gives the value $k_{\mathrm{B}} T_{c}=J / 0.22$ and Onzager's exact solution for $D=2$ Ising model leads to $k_{\mathrm{B}} T_{c}=$ $J / 0.44$. The numerical values of initial and canonical coefficients of LandauGinsburg Hamiltonian are equal to:

$$
\begin{array}{llll}
a_{4}=1 / 3, & b=1, & \alpha=1 & \\
a_{4}=0.98, & b=1, & \alpha=0.68 & \text { - initial }, \\
\text { canonical },
\end{array}
$$

where

$$
\alpha=\lim _{\tau \rightarrow 0} \frac{a_{2}}{\tau} .
$$

Where the Ginsburg number and correlation radius amplitude corresponding to (68) are:

$$
\begin{array}{lllll}
\mathrm{Gi}= & 0.1 & , \quad r_{0}= & \mathrm{c} & - \text { initial, } \\
\mathrm{Gi}= & 0.5, & r_{0}= & 1.2 \mathrm{c} & \text {-canonical, }
\end{array}
$$

where $c$ is lattice parameter. The situation becomes more complex for simple liquids which are described with help of mvdW equation. In this case the 
coefficients $\lambda_{k}$ are the functions of both the temperature and the external field. The use of expansions

$$
\gamma_{n}=\sum \gamma_{n}^{i_{1}, i_{2}} \tau^{i_{1}} h^{i_{2}}
$$

is not satisfactory because they converge in a small vicinity of $\tau^{*}, h^{*}$. That is why the solutions were obtained by numerical method. In order to calculate the coefficients $a, b, c$ in (7), (8) and (11) the standard formulas [8] were used:

$$
\begin{aligned}
a & =-2 \pi \sigma^{3} \int_{\sigma}^{\infty} \Phi(r) r^{2} \mathrm{~d} r \\
b & =\frac{2 \pi \sigma^{3}}{3} \\
c & =\frac{16 \pi^{3} \sigma^{3}}{3} \int_{\sigma}^{2 \sigma} \Phi(r)\left[1-\frac{3 r}{4 \sigma}+\frac{1}{16}\left(\frac{r}{\sigma}\right)^{3}\right] r^{2} \mathrm{~d} r,
\end{aligned}
$$

where $\Phi(r)$ is an intermolecular potential, $\sigma$ is hard core diameter. In (69) the following testing potentials:

$$
\begin{aligned}
& \Phi_{1}=-\epsilon\left(\frac{\sigma}{r}\right)^{6} \\
& \Phi_{2}=-4 \epsilon\left[\left(\frac{\sigma}{r}\right)^{6}-\left(\frac{\sigma}{r}\right)^{12}\right], \\
& \Phi_{3}=-\frac{27}{4} \epsilon\left[\left(\frac{\sigma}{r}\right)^{6}-\left(\frac{\sigma}{r}\right)^{9}\right],
\end{aligned}
$$

were substituted instead of $\Phi(r)$. The calculations of different parameters and its comparison with experimental ones were made for Ar and Xe. The parameters $\epsilon$ and $\sigma$ were put respectively:

$$
\epsilon / k_{\mathrm{B}}=119.8 K, \quad \sigma=3.405 \AA, \quad \epsilon / k_{\mathrm{B}}=225.3 K, \quad \sigma=4.04 \AA .
$$

The obtained data are presented in table 2 . Initial values of critical temperature and pressure were calculated with the help of (11) and (12). The roots of (62) were found by the dichotomy method in the vicinity of the initial critical point. There was used one iteration for calculating $\Gamma_{n}$ like in the case of Ising model. It appeared that the root satisfying all required conditions exists only for mvdW equation with Lenard-Jones and 6-9 potentials. In other cases the poor convergency properties of the initial equation of state is the cause of the nonexistence of the root. It is important that prime values of the critical point coordinates were close to the experimental ones. In this case the applying of the canonical formalism might improve the theoretical results. The fluctuation terms should be also taken into account to reduce the difference in the coordinates of the critical point. The same scheme can be applied to other equations of state including the empiric ones which describe the properties of liquids. The respective results will be the subject of other paper. The results in table 2 obtained for $\left.\lambda_{4}(T)\right|_{T=T_{0}}$, where $T_{0}$ is prime value of the critical temperature and the shift of the order parameter $\left(\psi=\phi-\frac{a_{3}}{3 a_{4}}\right)$ has been done. 


\section{Equation of state for simple liquid}

Constructing of the equation of state near the critical point on the basis of the canonical formalism allows one to take into account both the most general properties of fluctuations and individual features of different liquids and mixtures. This possibility is predetermined by the structure of the effective Hamiltonian and explicit form of its coefficients. The scale-invariant theory of fluctuations is known to lead to general expression for the singular part of the free energy:

Table 2. Some critical point parameters.

\begin{tabular}{|c|r|r|r|r|r|r|r|}
\hline & & vdW & vdW & vdW & mvdW & mvdW & mvdW \\
\hline \multirow{2}{*}{$T_{0}, K$} & $\mathrm{Ar}$ & 94.7 & $6-9$ & $6-\infty$ & $6-12$ & $6-9$ & $6-\infty$ \\
\cline { 2 - 8 } & $\mathrm{Xe}$ & 178.0 & 133.5 & 119.8 & 174.0 & 123.5 & 244.6 \\
\hline \multirow{2}{*}{$P_{0}$, bar } & $\mathrm{Ar}$ & 19.8 & 14.9 & 25.0 & 327.1 & 232.2 & 459.8 \\
\cline { 2 - 8 } & $\mathrm{Xe}$ & 22.2 & 16.7 & 28.1 & 91.4 & 54.1 & 131.3 \\
\hline$r_{0} \times 10^{8}, c m$ & $\mathrm{Ar}$ & 1.2 & 0.93 & 1.4 & 1.4 & 1.2 & 1.4 \\
\cline { 2 - 8 } & $\mathrm{Xe}$ & 1.5 & 1.1 & 1.7 & 1.7 & 1.5 & 1.9 \\
\hline $\mathrm{Gi}$ & & 2.0 & 5.0 & 0.4 & 0.02 & 0.03 & 0.01 \\
\hline \hline \multirow{2}{*}{$T_{c}^{c a n}, K$} & $\mathrm{Ar}$ & - & - & - & 158.6 & 190.5 & - \\
\cline { 2 - 8 } & $\mathrm{Xe}$ & - & - & - & 298.8 & 358.2 & - \\
\hline \multirow{2}{*}{$P_{c}^{c a n}, b a r$} & $\mathrm{Ar}$ & - & - & - & 51.8 & 57.1 & - \\
\cline { 2 - 8 } & $\mathrm{Xe}$ & - & - & - & 60.6 & 64.5 & - \\
\hline$r_{0}^{c a n} \times 10^{8}, \mathrm{~cm}$ & $\mathrm{Ar}$ & - & - & - & 1.2 & 1.0 & - \\
\cline { 2 - 8 } & $\mathrm{Xe}$ & - & - & - & 1.4 & 1.2 & - \\
\hline $\mathrm{Gi}$ & & & & & 0.05 & 0.08 & \\
\hline
\end{tabular}

Table 3. Experimental data.

\begin{tabular}{|c|r|r|c|}
\hline & $T_{c}, \mathrm{~K}$ & $P_{c}, \mathrm{bar}$ & $r_{0} 10^{8} \mathrm{~cm}$ \\
\hline $\mathrm{Ar}$ & 150.66 & 48.50 & 1.8 \\
\hline $\mathrm{Xe}$ & 289.80 & 58.40 & 2.1 \\
\hline
\end{tabular}

$$
F_{\text {sing }}=\left|h_{2}\right|^{2-\alpha} f_{s}\left(\frac{h_{1}}{h_{2}^{\beta+\gamma}}\right),
$$

where $h_{1}, h_{2}$ are the scaling fields, $\alpha, \beta, \gamma$ are the critical exponents for the specific heat, the equation of state and susceptibility, respectively, $f_{s}(x)$ is the universal scaling function. According to (33) $h_{1}$ and $h_{2}$ can be approximated by the coefficients of the canonical form for the Hamiltonian. As a result, in the framework of the canonical formalism the following form for the equation of state holds

$$
\langle\psi\rangle=\left|a_{2}\right|^{\beta} g_{s}\left(\frac{h_{\psi}}{a_{2}^{\beta+\gamma}}\right), \quad g_{s}(x)=f_{s}^{\prime}(x) .
$$


Here the brackets $\langle\ldots\rangle$ designates the averaging over the volume of the correlation sphere $\left(\propto r_{c}^{3}\right)$. In particular the binodal equation including the additional Wegner's term [19] is as following:

$$
\langle\psi\rangle_{\text {bin }}= \pm\left|a_{2}^{*}\right|^{\beta}\left(1+b_{2}\left|a_{2}^{*}\right|^{\Delta}+\ldots\right),
$$

where $a_{2}^{*}=\left.a_{2}\right|_{h_{\psi}=0}$. Its "liquid" and "gas" branches in variables $\left(\psi, a_{2}^{*}\right)$, as it is clear from (72), are absolutely symmetric. But this symmetry disappears if we return to the initial ("laboratory") variables $(\phi, \tau)$. Indeed, using (34) - (36) one can get:

$$
\langle\phi\rangle=\alpha_{0}+\langle\psi\rangle+\frac{1}{2} \Gamma_{2}\left\langle\psi^{2}\right\rangle+\ldots
$$

Since (see [1])

$$
\left\langle\psi^{2}\right\rangle=\langle\psi\rangle^{2}+\left|a_{2}\right|^{1-\alpha} l_{s}\left(\frac{h_{\psi}}{a_{2}^{\beta+\gamma}}\right),
$$

where function $l_{s}(x)$ is inverse to $g_{s}(x)$, therefore

$$
\begin{aligned}
\langle\phi\rangle=\alpha_{0} & +\left|a_{2}\right|^{\beta} g_{s}\left(\frac{h_{\psi}}{a_{2}^{\beta+\gamma}}\right)+ \\
& +\frac{1}{2} \Gamma_{2}\left[\left|a_{2}\right|^{2 \beta} g_{s}^{2}\left(\frac{h_{\psi}}{a_{2}^{\beta+\gamma}}\right)+\left|a_{2}\right|^{1-\alpha} l_{s}\left(\frac{h_{\psi}}{a_{2}^{\beta+\gamma}}\right)\right]+\ldots,
\end{aligned}
$$

and

$$
\begin{aligned}
\langle\phi\rangle_{\text {bin }}=\alpha_{0} & \pm g_{s}(0)\left|a_{2}^{*}\right|^{\beta}\left(1+b_{2}\left|a_{2}^{*}\right|^{\Delta}+\ldots\right)+ \\
& +\frac{1}{2} \Gamma_{2}\left[g_{s}^{2}(0)\left|a_{2}^{*}\right|^{2 \beta}+l_{s}(0)\left|a_{2}^{*}\right|^{1-\alpha}\right]+\ldots
\end{aligned}
$$

Usually equation (76) is written as two separate equations:

$$
\begin{aligned}
& \frac{1}{2}\left(\langle\phi\rangle_{\text {bin }}^{+}-\langle\phi\rangle_{\text {bin }}^{-}\right)=g_{s}(0)\left|a_{2}^{*}\right|^{\beta}\left(1+b_{2}\left|a_{2}^{*}\right|^{\Delta}+\ldots\right)+\ldots \\
& \frac{1}{2}\left(\langle\phi\rangle_{\text {bin }}^{+}+\langle\phi\rangle_{\text {bin }}^{-}\right)=\alpha_{0}+\frac{1}{2} \Gamma_{2}\left[g_{s}^{2}(0)\left|a_{2}^{*}\right|^{2 \beta}+l_{s}(0)\left|a_{2}^{*}\right|^{1-\alpha}\right]+\ldots
\end{aligned}
$$

The second equation leads to the rectilinear diameter singularity [1]. The temperature dependence of coefficients

$$
\begin{aligned}
& \alpha_{0}=\alpha_{01} \tau+\alpha_{02} \tau^{2}+\ldots \\
& \Gamma_{2}=\Gamma_{20}+\Gamma_{21} \tau+\Gamma_{22} \tau^{2}+\ldots \\
& a_{2}^{*}=a_{21} \tau+a_{22} \tau^{2}+\ldots
\end{aligned}
$$

is determined by the formulas (34)-(36). For the fluids far away from the critical point described by the same equation of corresponding states the coefficients $\alpha_{0}, \Gamma_{2}$ and $a_{2}^{*}$ coincide. In particular, for the simple liquids described by mvdW equation with Lenard - Jones potential the coefficients (78) are as following:

$$
\begin{aligned}
& \alpha_{0}=-0.19 \tau \\
& \Gamma_{2}=0.12 \tau \\
& a_{2}^{*}=-3.1 \tau
\end{aligned}
$$


The coefficients $b_{2}$ and $g_{s}(0), l_{s}(0)$ take universal values and can be estimated from the experimental data with the help of formulas given above. For example, by applying the mvdW equation to the experimental data we get for the rectilinear diameter of $\mathrm{Ar}$ and Xe the following expression we get:

$$
\begin{gathered}
\frac{1}{2}\left(\langle\phi\rangle_{\text {bin }}^{+}+\langle\phi\rangle_{\text {bin }}^{-}\right)=-0.19 \tau+0.1|\tau|^{2 \beta}+0.4|\tau|^{1-\alpha}+\ldots \\
l_{s}(0)=\frac{0.4}{0.5\left|a_{21}\right|^{1-\alpha} \Gamma_{20}} \simeq 2.4, \quad g_{s}(0)=\sqrt{\frac{0.1}{0.5\left|a_{21}\right|^{2 \beta} \Gamma_{20}}} \simeq 0.89
\end{gathered}
$$

for Lenard - Jones potential and

$$
\begin{gathered}
\frac{1}{2}\left(\langle\phi\rangle_{\text {bin }}^{+}+\langle\phi\rangle_{\text {bin }}^{-}\right)=-1.16 \tau+0.06|\tau|^{2 \beta}+1.1|\tau|^{1-\alpha}+\ldots \\
l_{s}(0)=\frac{1.1}{0.5\left|a_{21}\right|^{1-\alpha} \Gamma_{20}} \simeq 6.5, \quad g_{s}(0)=\sqrt{\frac{0.06}{0.5\left|a_{21}\right|^{2 \beta} \Gamma_{20}}} \simeq 0.68
\end{gathered}
$$

for 6-9 potential.

We would like to stress the fact that in our approach the asymmetry of coexistence curve appears as a consequence of inverse transformation from the canonical variables to the initial ones. In more standard considerations [20] this effects arises due to addition of the odd terms in to the effective Hamiltonian. The expression (74) can be interpreted as an expansion of the initial order parameter over the basis of the strongly fluctuating fields [1]. This allows us to consider the canonical formalism as the approach generalizing the heuristic arguments developed in [1]. Note that with the help of formula (75) one can qualitatively explain the differences of gravitational effects in simple [2] and molecular [21] liquids.

Using the canonical formalism the different flattening of closed lamination curves in binary mixtures with hydrogen bonds near upper $\left(T_{u}\right)$ and lower $\left(T_{l}\right)$ critical points can be explained [22]. In this case the coefficients $a_{21}\left(T_{l}\right)$ and $a_{21}\left(T_{u}\right)$ responsible for flattening can be estimated with the help of some model [23] and are quite different.

In the same way one can consider the connection between the asymmetry of equation of state and the choice of an order parameter both in simple liquids and in multicomponent mixtures.

\section{Conclusion}

In this paper the main elements of the canonical formalism and it's applications to Ising model and simple liquids were presented. We would like to accentuate that the proposed approach is not only formal procedure leading to more clear mathematical consideration but also gives new physical results. Among them we note: the rigorous definition of the initial order parameter; generalized temperature and external field conjugated to it, the nontrivial renormalization of initial values for many critical parameters, more correct formulation of RG-theory, the consecutive explanation of equation of state asymmetry etc. These additional possibilities of the theory are connected with the more accurate account of small-range fluctuations. Due to the using of the canonical formalism we are able to separate the effects raised by the long-wave fluctuations which have general character and the individual properties of the system. As a result one can evaluate the theoretical 
constants like $g_{s}(0)$ and $l_{s}(0)$ and so on. Here we only considered the consequences of local canonical transformation. We think that wider possibilities are connected with further development of the canonical formalism in which the quasilocal canonical transformations reducing the effective Hamiltonian to the canonical forms like Ginsburg-Landau Hamiltonian will play the key role. New applications of the proposed approach to electrolytes, polymer and micellar solutions as well as to conductor-insulator transition in the vicinity of the critical point are very attractive too.

\section{Acknowledgements}

The authors are grateful to never-to-be-forgotten D.N.Zubarev for encouragement of the work on its primary stage. We are thankful to V.G.Morozov, S.V.Peletminskii for discussion of the topics. We also thank to I.Stasyuk, M.Kozlovskii and Yu.Holovatch for the possibility of discussing the results on the seminar of ICMP. This work has been done due to financial support of ISSEP (grants No PSU 052077 - V.K., No PSU 062032 - N.M.)

\section{References}

[1] Patashinsky A.Z., Pokrovsky V.L. Fluctuation theory of the critical phenomena. Moscow, Nauka, 1982

[2] Anisimov M.A. Critical phenomena in liquids and liquid crystals. Moscow, Nauka, 1987

[3] Wilson K.G. Renormalization group and critical phenomena 1. Renormalization group and Kadanoff scaling picture. // Phys. Rev. B, 1971, vol. 4, No 9, p. 3174-3183.

[4] Wilson K.G. Renormalization group and critical phenomena 2. Phase space cell analysis of critical behaviour. // Ibid., p. 3184-3205.

[5] Arnold V.I. Singularity theory. Selected Papers. London Math. Soc., Lecture Notes, vol. 53. Cambridge University Press, 1981.

[6] Guillou Le J.C., Zinn-Justin J. Critical exponents from field theory. // Phys. Rev. B, vol. 21, No 7, p. 3976-3998.

[7] Izyumov Yu.A., Skryabin Yu.N. Statistical mechanics of magnetic-ordered systems. Moscow, Nauka, 1987

[8 Croxton C.A. Liquid state physics. Cambridge University Press, 1974.

[9] Hubbard J., Schofield P. Wilson theory of a liquid-vapour critical point. // Phys. Lett., 1972, vol. 40A, No 3, p. 245-246.

[10] Yukhnovskii I.R. Phase transitions of the second order. Collective variable method. Singapore, World Scientific, 1987.

[11] Yukhnovskii I.R., Holovko M.F. The statistical theory of classical equilibrium systems. Kiev, Naukova Dumka, 1980.

[12] Wilson K.G. Renormalization group: Critical phenomena and Kondo problem. // Rev. Mod. Phys., 1975, vol. 47, No 4, p. 773-840.

[13] Poston T., Stewart I.N. Catastrophe theory and its applications. Surveys and reference works in math. 2. London, Pitman, 1978.

[14] Koulinskii V.L., Malomuzh N.P., Veytsman B.A. Canonical formalism and renormalization group. // Proc. Int. Conf. "Renormgroup-91", Dubna, 3-6 Sept., p. 230-242.

[15] Koulinskii V.L., Malomuzh N.P. Canonical formalism of the critical phenomena description. // Proc. Int. Conf. "Physics in Ukraine", Kiev, 22-27 June 1993, p. $72-75$.

[16] Koulinskii V.L. Canonical formalism for description of critical phenomena in simple systems. // Ukr. Fiz. Zhurn., 1993, vol. 38, No 12, p. 1872-1880.

[17] Gell-Mann M., Low F.E. Quantum electrodynamics of small distances. // Phys. Rev., 1954, vol. 95, No 5, p. 1300-1312. 
[18] Vakarchuk I.A, Rudavskii Yu.K., Holovatch Yu.V. // Phys. Manypart. Syst. (Ukr.), 1983, vol. 4, p. 44-59.

[19] Wegner F.J. Corrections to scaling laws. // Phys. Rev. B., 1972, vol. 5, No 5, p. $4529-4536$.

[20] Nicoll J.F. Critical phenomena in liquids: Asymptotic Landau-GinsburgWilson model. // Phys. Rev. B., 1981, vol. 24, No 4, p. 2203-2220.

[21] Balevicuis V., Weiden N., Weiss A. Deitron NMR relaxation, phase diagrams, and isotope effects in liquid mixtures of tetra hydro fura $/ D_{2} O /$ salt. // Ber. Bunsenges, Phys. Chem., 1994, vol. 98, No 6, p. 785-792.

[22] Malomuzh N.P., Veytsman B.A. Properties of binary and ternary mixtures in the vicinity of double critical points. // Phys. Lett. A, 1989, vol. 136, p. 239-244.

[23] Koulinskii V.L., Malomuzh N.P. Nature of the asymmetry of the equation of state near critical points in a liquid with hydrogen bonding. // J. Mol. Struc., 1996, vol. 381, No 1-3, p. 199-206.

\title{
КАНОНІЧНИЙ ФОРМАЛІЗМ ДЛЯ ОПИСУ КРИТИЧНИХ ЯВИЩ В СИСТЕМАХ, ЩО $\mathbf{E}$ ІЗОМОРФНИМИ ДО ПРОСТИХ РІДИН
}

\author{
В.Л.Колінський, Н.П.Маломуж
}

Констатуються основні елементи канонічного формалізму для опису критичних явищ у системах, що є ізоморфними до моделі Ізінга. Грунтовно досліджується процедура приведення ефективного гамільтоніана до канонічної форми з численними наслідками такого перетворення. Розглядаються зсув фіксованої точки та критичних показників. 